\title{
The RAND-36 Health Survey 1.0: Translation, Reliability, Cross-Cultural Adaptation and Validation of the Gujarati Version
}

\author{
Hemang Jani ${ }^{1}$, Gauravi Dhruva ${ }^{2}$, Dinesh Sorani ${ }^{3}$, Yagnik Dave ${ }^{4}$ \\ ${ }^{1}$ Inch. Principal/Associate Professor, Harivandana Physiotherapy College, Rajkot, Gujarat, India. \\ ${ }^{2}$ HOD, Pathology Department, PDU Medical College, Rajkot. \\ ${ }^{3}$ Principal, Govt. Physiotherapy College, Jamnagar. \\ ${ }^{4}$ Assistance Professor, Faculty of Physiotherapy, Marwadi University, Rajkot. \\ Corresponding Author: Hemang Jani
}

\begin{abstract}
Background: The Short Form 36 Item Survey is the most typically used instrument for assessing health-related quality of life. ${ }^{1}$ Two identical versions of the initial instrument are currently available: the general public domain, licensefree RAND-36, and also the commercial SF $36 .{ }^{2}$ RAND 36 don't seem to be available within the Gujarati language. The aim of this study was to translate and culturally adapt the RAND 36 into the Gujarati language and measure its reliability and validity.
\end{abstract}

Methods: According to the guidelines by the International Quality of Life Assessment project, a test of item-scale correlation, a sequence of translation, and validation were implemented for the translation of the Gujarati version of the RAND-36. Following pilot testing, the English and the Gujarati versions of the RAND-36 were administered to a random sample of 120 apparently healthy individuals to test validity and 96 respondents completed the Gujarati RAND-36 again after two weeks to test reliability. Data were analyzed using one-way analysis of variance, multi-trait scaling analysis, one-way analysis of variance, Pearson's product-moment correlation analysis, and IntraClass Correlation (ICC) at $\mathrm{p}<0.05$

Results: The median Cronbach's alphas for the Gujarati RAND-36 in multiple subgroups exceeded 0.70 for every scale except one. Two of the English RAND-36 scales had median Cronbach's alphas that exceeded 0.70 ; the rest exceeded 0.50. Test-retest correlations were found statistically significant for both versions.
Product-moment correlations to test the equivalence of the corresponding Gujarati and English versions of the RAND-36 ranged from 0.73 to 0.92 . The Gujarati version of the RAND36 has high internal consistency (Cronbach's $\alpha=0.809$ ) and test-retest reliability (Intra-class correlation coefficient $=0.746,95 \%$ CI: 0.58 , 0.94).

Conclusions: The Gujarati version of the RAND-36 performed well and the findings suggest that it is a reliable and valid measure of health-related quality of life among the general Gujarati population.

Keywords: RAND-36, cross-cultural translation, quality of life, health status assessment, Gujarati.

\section{INTRODUCTION}

Health is defined as complete mental, physical and social well-being with the absence of disease. The RAND 36 Health Survey is a quality of life Questionnaire. It consisted of the same items included in the Medical Outcomes Study 36 Items Short-Form Health Survey (SF-36). Both tools came out from work begun at RAND in 1984 as part of the Medical Outcomes Study (MOS) and include multi-item scales that assess eight health concepts: physical functioning, bodily pain, role limitations due to physical health problems, role limitations due to emotional problems, mental health (emotional well-being), social functioning, 
vitality (energy/fatigue), and general health. The only difference between the measures is the scoring algorithm applied when calculating two of the scale scores (bodily pain, general health), which is independent of the evaluation process. Hence, for the comparison with published general population norms, SF-36 scoring was used. There is a growing body of evidence that the SF-36 can be successfully translated, validated, and normalized for use in other countries. ${ }^{3}$ This article describes the administration and evaluation of a new Gujarati translation of the RAND-36 in a sample of Gujarati People. The translation process that initiated this work was conducted in agreement with a commonly accepted cross-cultural adaptation methodology. ${ }^{4,6}$

\section{METHODS}

Sample

This study was conducted at Harivandana Physiotherapy clinic Rajkot, Gujarat. India. A convenience sample was selected from several departments at Harivandana Physiotherapy clinic Rajkot. This sample was selected by asking each of the departments to generate a list of bilingual (Gujarati and English) Gujarati employees who were willing to participate in the study. Participants were selected from each department to work as coordinators with the second author. After obtaining complete lists of participants from the departments, each individual was assigned randomly to one of six groups. 60 subjects were assigned to each of Groups A, B, and $\mathrm{C}$ and 60 subjects were assigned to each of Groups D, E, and F. After taking written informed concern all the participants were enrolled in this study.

\section{Data Collection:}

Data were collected by distributing the English and/or Gujarati versions of the RAND-36 to the participants through the coordinators. Cover letters that explain the purpose of the study were provided. Additional questions were included to gather demographic information (i.e, gender, age, and education), and the questionnaires were coded so that nonrespondents could be contacted. The list that linked the questionnaire codes to specific individuals can be accessed by only the second author. These questionnaires were administered twice (time $1=$ initial; time 2 $=$ retest $)$ as follows:

Group A. subjects completed the English version first then the Gujarati version. A sealed envelope containing both versions to the subjects was distributed by the assigned coordinator.

The subjects were given instruction to complete both questionnaires as follows:

(1) Complete the English questionnaires put it in the envelope provided and close the envelope,

(2) Complete the Gujarati questionnaire, put it in the other envelope and close the envelope, and

(3) Return both envelopes to the assigned coordinator.

Aside from the order of administration, instructions for Groups B through $\mathrm{F}$ were as discussed for Group A.

Group B. Participants completed the Gujarati version first, immediately followed by the English version.

Group C. Participants completed the English version followed by the Gujarati version after a 2-week interval.

Group D. Participants completed the Gujarati version followed by the English version after a 2 -week interval.

Group E. Participants completed the Gujarati version two times ( 2 weeks apart).

Group F. Participants completed the English version two times (2 weeks apart).

\section{Scoring:}

All scales were converted linearly to a 0 to 100 possible variation of scores, with 0 and 100 representing the least and most favorable health state, respectively. All scores indicate the percent of the total possible score for that scale. The RAND 36 was scored according to the methodology used for the published norms for the SF 36.7 
in order to compare scores from this sample to those from the Indian general population. For other detail examination, the pain and therefore scoring of general health perception scales followed the RAND 36 recommendations. 1

\section{Data Analysis:}

Statistical analyses were conducted using SPSS 20.

Descriptive Statistics. The average ages, percentage of men and women, and degree of education of respondents in each category were determined.

\section{Internal Consistency Reliability.}

Cronbach's coefficient alpha was used to estimate internal consistency reliability for each of the administrations of Gujarati and English versions. ${ }^{8}$

\section{Test-Retest Reliability.}

To assess test-retest reliability during a 2-week interval, Pearson productmoment correlations were computed between initial and retest administrations for both Group E and Group F.

\section{Equivalent Forms Reliability.}

Pearson product-moment correlation coefficients were computed to evaluate equivalent forms reliability between the Gujarati and English versions administered on the same day (Groups A and B). Scale Score Means. Scale score means were computed for the Gujarati and English versions to determine if central tendency varied by version.

\section{RESULTS}

Subjects of the 120 subjects randomly assigned to the six study groups, $96(78 \%)$ individuals returned their questionnaires. Of these, 24 individuals were excluded from the study because they had missing data for one or more items. Thus, the analytic sample size was 96 . The mean age of the subjects was $34.9+7.0$ years, with a range of 25 to 55 years. Seventy-eight percent of the subjects were male, and $52 \%$ were college graduates.
ANOVA test was applied for comparing the age of the six groups found no statistically significant difference $[\mathrm{F}(5,358)=0.929, \mathrm{P}$ $>0.05]$.

\section{Internal Consistency Reliability:}

Gujarati Version Initial Administration. Alpha reliability coefficients for the initial administration of the Gujarati version (Groups B, D, and E, $n$ $=48$ ) ranged from 0.60 (general health) to 0.87 (physical functioning). Reliability coefficients exceeded 0.70 , except for the general health scale which follows Nunnally's standard of acceptable reliability for group-level studies. ${ }^{9}$

\section{Retest Administration:}

Alpha coefficients for retest administrations (Groups $\mathrm{A}, \mathrm{C}$, and $\mathrm{E}, \mathrm{n}=$ 48) ranged from 0.57 (general health) to 0.88 (physical functioning) and were 0.70 or higher for all scales except for general health.

\section{Internal Consistency Reliability: English Version Initial Administration.}

Alpha coefficients for the initial administrations of the English version (Groups $\mathrm{A}, \mathrm{C}$, and $\mathrm{F}, \mathrm{n}=48$ ) ranged from 0.56 (role limitations-physical, social functioning) to 0.89 (physical functioning). Alpha coefficients for four other scales were less than 0.70 [Role limitations-emotional (0.66), Mental health (0.68), Vitality (0.58), General health (0.61)].

Retest Administration. Alpha coefficients for the retest administrations (Groups B, D and $\mathrm{F}, \mathrm{n}=48$ ) ranged from 0.60 (social functioning) to 0.90 (physical functioning). Alpha coefficients for two other scales were less than 0.70 (general health (0.68), vitality (0.61)). Test-Retest Reliability Two-week test-retest product-moment correlations were all statistically significant and ranged from 0.29 (bodily pain) to 0.80 (mental health) for the Gujarati version $(n=48)$ and from 0.46 (role limitations-physical) to 0.77 (general health) for the English version ( $\mathrm{n}=$ 48). Equivalent-Forms Reliability 
Hemang Jani et.al. The RAND-36 health survey 1.0: translation, reliability, cross-cultural adaptation and validation of the Gujarati version.

Equivalent-forms product-moment correlations between corresponding scales for those administered the English version immediately followed by the Gujarati version $(\mathrm{n}=48)$ ranged from 0.78 (social functioning) to 0.91 (mental health). Correlations for those administered the Gujarati version immediately followed by the English version $(\mathrm{n}=48)$ ranged from 0.73 (vitality) to 0.92 (mental health).

\begin{tabular}{|c|c|c|c|}
\hline Scale & $\begin{array}{l}\text { Number of } \\
\text { Items }\end{array}$ & $\begin{array}{l}\text { Initial Gujarati Administration } \\
(n=48)\end{array}$ & $\begin{array}{l}\text { Initial English Administration } \\
(\mathrm{n}=\mathbf{4 8})\end{array}$ \\
\hline Physical functioning & 10 & $87.3(17.1)$ & $82.4(20.6)$ \\
\hline Role limitations due to physical health & 4 & $84.3(30.3)$ & $90.3(19.4)$ \\
\hline $\begin{array}{l}\text { Role limitations due to emotional } \\
\text { problems }\end{array}$ & 3 & $76.3(35.2)$ & $82.3(29.3)$ \\
\hline Energy/fatigue & 4 & $67.6(16.8)$ & $68.1(16.8$ \\
\hline Emotional well-being & 5 & $75.2(16.1)$ & $75.6(15.6)$ \\
\hline Social functioning & 2 & $82.0(19.3)$ & $79.0(20.7)$ \\
\hline Pain & 2 & $80.3(21.9)$ & $81.1(19.0)$ \\
\hline General Health & 5 & $71.1(16.1$ & $74.5(15.1)$ \\
\hline
\end{tabular}

\section{DISCUSSION}

The median internal consistency reliability coefficients for all administrations (Groups A, C, and E) of the Gujarati version exceeded 0.70 for every scale except for general health (median alpha $=0.59$ ). The median internal consistency reliability coefficients for all administrations (Groups B, D, and F) of the English version exceeded 0.50 . Therefore, the results of this study provide support for the reliability of the Gujarati version and are consistent with previous reliability estimates reported for the English version.

Both the Gujarati and English versions tended to have internal consistency reliability coefficients equal to or above the acceptable standards for group comparisons; ${ }^{\prime} 10$ however, the Gujarati version had higher median values than the English version. Although all participants were bilingual, their mother language was Gujarati.

As a result, it is likely that the participants had a better understanding of the Gujarati version than the English version, leading to more internally consistent responses. In addition, the Gujarati version was adapted to the Gujarati culture, whereas the English version was developed for the dominant US culture. Test-retest correlations during a 2-week time interval were similar for the English and Gujarati versions. The size of the correlations indicates a noteworthy degree of variation between initial and retest administrations. The study timing may have contributed to the fluctuation in scale scores.

Many Gujarati citizens suffer from Covid-19 and related symptoms during the time of year the study were conducted. Because of attainable actual changes, testretest reliability estimates need to be evaluated with caution, especially when studying a dynamic process such as health status. $^{11}$ The equivalence of the corresponding English and Gujarati versions of the RAND 36 scale was assessed using correlations.

Product moment correlations were ranging from 0.73 to 0.92 between corresponding scales. These results provide strong support for the equivalence of the Gujarati and English versions. The results found by this study indicate the good reliability of a Gujarati version of the RAND-36 and its equivalence with the English version.

\section{CONCLUSIONS}

The Gujarati version of the RAND36 performed well and the findings suggest that it is a reliable and valid measure of health-related quality of life among the general Gujarati population. 
Hemang Jani et.al. The RAND-36 health survey 1.0: translation, reliability, cross-cultural adaptation and validation of the Gujarati version.

ACKNOWLEDGMENT: The Authors are thankful to Trustees of Harivandana Physiotherapy College, Rajkot for continuous support during study.

\section{Conflict of Interest: None}

\section{Source of Funding: None}

\section{REFERENCES}

1. Hays RD, Sherbourne CD, Mazel RM. The RAND 36-Item Health Survey 1.0. Health Econ 1993;2:217.

2. Ware JE Jr, Sherbourne CD. The MOS 36Item Short-Form Health Survey (SF-36): I. Conceptual framework and item selection. Med Care 1992;30:473.

3. Ware JE Jr, Gandek BL, Keller SD, et al. Evaluating instruments used crossnationally: Methods from the IQOLA project. In: Spilker B, ed. Quality of life and pharmacoeconomics in clinical trials, second edition. Philadelphia, PA: Lippincott-Raven Publishers, 1996:681.

4. Guillemin F, Bombardier C, Beaton D. Cross-cultural adaptation of quality of life measures: Literature review and proposed guidelines. J Clin Epidemiol 1993; 46:1417.

5. Alabdulmohsin SA, Coons SJ, Draugalis JR, Hays RD. Translation of the RAND 36-Item
Health Survey 1.0 (aka SF-36) into Arabic. Santa Monica, CA: RAND, P-7995, 1997.

6. Medical Outcomes Trust. SF-36 scoring man-ual for English language adaptations: Australia/New Zealand, Canada, United Kingdom. Boston, MA: Medical Outcomes Trust, 1994.

7. Ware JE Jr, Snow KK, Kosinski M, Gandek B. SF-36 Health Survey: Manual and interpretation guide. Boston, MA: Nimrod Press, 1993.

8. Cronbach LJ. Coefficient alpha and the internal structure of tests. Psychometrika 1951;16:297.

9. Nunnally JC. Psychometric theory, second edition. New York, NY: McGraw-Hill, 1978.

10. Helmstadter GC. Principles of psychological measurement. New York: Appleton-Century-Crofts, 1964.

11. Coons SJ, Kaplan RM. Assessing healthrelated quality of life: Application to drug therapy. Clin Ther 1992;14:850.

How to cite this article: Jani $\mathrm{H}$, Dhruva G, Sorani D et.al. The RAND-36 health survey 1.0: translation, reliability, cross-cultural adaptation and validation of the Gujarati version. International Journal of Science \& Healthcare Research. 2021; 6(4): 52-56. DOI: https:// doi.org/10.52403/ijshr.20211008 